\title{
Article \\ Application of Gamma Ray-Responsive Genes for Transcriptome-Based Phytodosimetry in Rice
}

\author{
Jin-Hong Kim ${ }^{1,2}, * \mathbb{D}$, Kwon Hwangbo ${ }^{1}$, Eujin Lee ${ }^{1}$, Shubham Kumar Dubey ${ }^{1,2}$, Moon-Soo Chung ${ }^{1}$, \\ Byung-Yeoup Chung ${ }^{1}$ and Sungbeom Lee ${ }^{1,2}$
}

1 Advanced Radiation Technology Institute, Korea Atomic Energy Research Institute, 29 Geumgu-gil, Jeongeup-si 56212, Korea; kwonhb@kaeri.re.kr (K.H.); dldbwls10@daum.net (E.L.); shubham26@kaeri.re.kr (S.K.D.); mschung@kaeri.re.kr (M.-S.C.); bychung@kaeri.re.kr (B.-Y.C.); sungbeom@kaeri.re.kr (S.L.)

2 Department of Radiation Science and Technology, University of Science and Technology, 217 Gajeong-ro, Yuseong-gu, Daejeon 34113, Korea

* Correspondence: jhongkim@kaeri.re.kr; Tel.: +82-63-570-3333

Citation: Kim, J.-H.; Hwangbo, K.; Lee, E.; Dubey, S.K.; Chung, M.-S.; Chung, B.-Y.; Lee, S. Application of Gamma Ray-Responsive Genes for Transcriptome-Based Phytodosimetry in Rice. Plants 2021, 10, 968. https:// doi.org/10.3390/plants10050968

Academic Editors: Khalid Meksem and Sibin Yu

Received: 22 March 2021

Accepted: 12 May 2021

Published: 13 May 2021

Publisher's Note: MDPI stays neutral with regard to jurisdictional claims in published maps and institutional affiliations.

Copyright: (c) 2021 by the authors. Licensee MDPI, Basel, Switzerland. This article is an open access article distributed under the terms and conditions of the Creative Commons Attribution (CC BY) license (https:/ / creativecommons.org/licenses/by/ $4.0 /)$.

\begin{abstract}
Transcriptome-based dose-response curves were recently applied to the phytodosimetry of gamma radiation in a dicot plant, Arabidopsis thaliana, as an alternative biological assessment of genotoxicity using DNA damage response (DDR) genes. In the present study, we characterized gamma ray-responsive marker genes for transcriptome-based phytodosimetry in a monocot plant, rice (Oryza sativa L.), and compared different phytodosimetry models between rice and Arabidopsis using gamma-H2AX, comet, and quantitative transcriptomic assays. The transcriptome-based doseresponse curves of four marker genes (OsGRG, OsMutS, OsRAD51, and OsRPA1) were reliably fitted to quadratic or exponential decay equations $\left(r^{2}>0.99\right)$. However, the single or integrated dose-response curves of these genes were distinctive from the conventional models obtained by the gamma-H2AX or comet assays. In comparison, rice displayed a higher dose-dependency in the comet signal and OsRAD51 transcription, while the gamma-H2AX induction was more dose-dependent in Arabidopsis. The dose-dependent transcriptions of the selected gamma-ray-inducible marker genes, including OsGRG, OsMutS, OsRAD51, and OsRPA1 in rice and AtGRG, AtPARP1, AtRAD51, and AtRPA1E in Arabidopsis, were maintained similarly at different vegetative stages. These results suggested that the transcriptome-based phytodosimetry model should be further corrected with conventional genotoxicity- or DDR-based models despite the high reliability or dose-dependency of the model. In addition, the relative weighting of each gene in the integrated transcriptome-based dose-response model using multiple genes needs to be considered based on the trend and amplitude of the transcriptional change.
\end{abstract}

Keywords: gamma radiation; phytodosimetry; rice; Arabidopsis; transcriptome; genotoxicity

\section{Introduction}

The nuclear accidents at Chernobyl and Fukushima have greatly deepened public concern about environmental contamination from artificial radionuclides in many countries, especially in those operating nuclear power plants. Environmental radioactive substances can be transferred into the human body through non-human biota that constitute the food chain in the ecosystem [1]. Therefore, it is necessary to develop a dedicated biological risk assessment and dosimetry model based on the relationship between radiation dose and its effects using a variety of reference animals and plants [2,3]. In this regard, plants are considered to be more suitable reference biota for environmental risk assessment due to their immobility compared with animals as well as the accumulated data regarding the biological effects of ionizing radiation in various plant species [4].

Conventional biological dosimetry (biodosimetry) of ionizing radiation has utilized the gold-standard methods of dicentric chromosome and micronucleus assays to estab- 
lish a dose-response curve based on chromosomal aberration since the 1960s [5-7]. In addition, $\gamma \mathrm{H} 2 \mathrm{AX}$ and single-cell gel electrophoresis (comet) assays have been adopted for biodosimetry to quantify the DNA damage response (DDR) associated with chromosomal aberration [8-11]. However, these conventional methods need to be complemented with more economic and efficient methods for plant biodosimetry (phytodosimetry) [4].

Radiation-specific transcriptomes are some of the most promising potential biomarkers that highlight the detrimental biological effects of ionizing radiation [12,13]. The degree of DNA damage can be quickly and accurately quantified by quantitative real time polymerase chain reaction (RT-qPCR) analysis [14]. Our recent study showed that an alternative transcriptome-based dose-response model for Arabidopsis thaliana, a dicot model plant, could be practically applied to phytodosimetry, the biological dosimetry of ionizing radiation in plants [4]. However, since there is a large difference between the genome in monocot and dicot plants [15-17], the availability of such transcriptome-based phytodosimetry needs to be further substantiated in monocot plants. Although DDR genes common to eukaryotic cells are preferentially considered for phytodosimetry, the radiation-responsive DDR genes of Arabidopsis may be not effective in rice (Oryza sativa), a monocot model plant. In addition, the coverage of phytodosimetry for environmental risk assessment of radioactive substances in the ecosystem will be expanded greatly by adding monocot plants. Despite a number of genetic studies in rice, until recently, radiation-responsive transcriptomic profiles had never been explored at the whole genome level after in planta exposure of monocot plants to ionizing radiation.

A recent report revealed the genome-wide radiation-responsive transcriptomic profiles in rice via RNA-seq analysis after gamma irradiation and suggested that the radiationresponsive transcriptomes in rice need to be functionally verified before being used for phytodosimetry due to the substantial difference in the expression of the DDR genes compared to those in Arabidopsis [18]. In this study, we selected several radiation-responsive marker genes, including DDR genes based on the rice RNA-seq data, and analyzed the dose-response curves of their transcriptomes for phytodosimetry in rice after gamma irradiation below tens of Gy. The transcriptomic changes in the marker genes after gamma irradiation were compared with the dose-dependent induction of DNA damages revealed by $\gamma \mathrm{H} 2 \mathrm{AX}$ and comet assays as dose-response models. The importance of using plant species-specific genes as well as those common to both monocot and dicot plants was discussed in terms of transcriptome-based phytodosimetry for the environmental risk assessment of ionizing radiation.

\section{Results and Discussion}

2.1. Dose-Response Curves of the DNA Damage Response (DDR) in Rice after Gamma Irradiation

Ionizing radiation, a potent genotoxic agent, causes physiological alterations as well as DNA damages including strand breaks, base modification and crosslinking [19-21]. Some photosynthesis-associated chlorophyll fluorescence parameters such as non-photochemical quenching and the photosynthesis performance index are known to be substantially decreased by gamma radiation below tens of Gy [22,23]. However, since there is no doseresponse model for such a dose range available based on plant physiology that includes photosynthesis, we performed $\gamma \mathrm{H} 2 \mathrm{AX}$ and comet assays to estimate the dose-dependency of DDR in rice upon exposure to various doses of gamma radiation from 3 to $200 \mathrm{~Gy}$, as previously reported in Arabidopsis [4]. When histone H2AX phosphorylation $(\gamma \mathrm{H} 2 \mathrm{AX})$, an initial response to induced DNA double strand breaks (DSBs), was evaluated by immunoblotting, the $\gamma \mathrm{H} 2 \mathrm{AX}$ protein was undetectable below $24 \mathrm{~Gy}$, but increased dosedependently from 24 to $200 \mathrm{~Gy}$ (Figure 1A). The dose-response of $\gamma \mathrm{H} 2 \mathrm{AX}$ was fitted to the linear-quadratic model:

$$
y=c+\beta x+\alpha x^{2}
$$


where $y$ is the response, $x$ is the radiation dose, $\mathrm{c}$ is the background induction level, $\beta$ is the linear component of the curve, and $\alpha$ is the quadratic portion of the curve. As a result, the dose-response equation of $\gamma \mathrm{H} 2 \mathrm{AX}$ induction as follows (Figure 1B):

$$
y=0.819+0.018 x-0.00003281 x^{2}\left(r^{2}=0.953\right)
$$
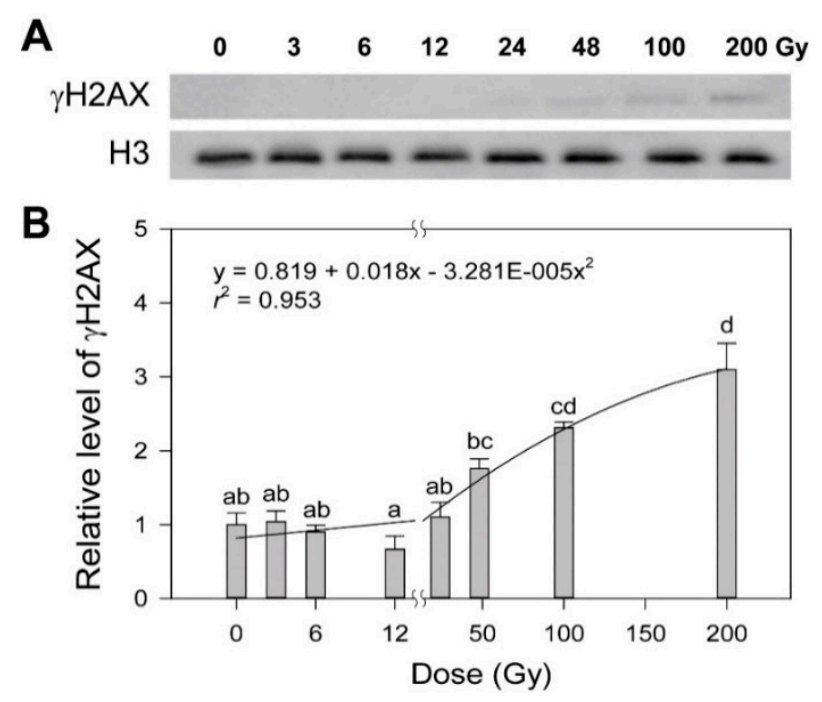

Figure 1. Induction of the $\gamma \mathrm{H} 2 \mathrm{AX}$ protein in rice irradiated with different doses of gamma radiation. The representative images (A) were obtained by Western blot analysis and subjected to regression for the quadratic dose-response curve (B). H3 was used as a loading control. Data are reported as the mean \pm standard error (SE) of three independent experiments. Different letters indicate significant differences at $p<0.05$ (one-way analysis of variance followed by Tukey's honestly significant difference test).

Although this equation represented a conventional dose-response curve for DDR in rice, it demonstrated relatively low confidence due to the lack of detectable $\gamma \mathrm{H} 2 \mathrm{AX}$ below 24 Gy, compared to that with $r^{2}=0.99$ in Arabidopsis [4].

Since DSBs are the most deleterious type of DNA damage but are rapidly recoverable by homologous recombination (HR) or non-homologous end-joining, they can be substantially detectable only within $1 \mathrm{~h}$ after irradiation [24-26]. The alkaline comet assay method measures various DNA damages including frank strand breaks, incomplete excision repair sites, alkali-labile sites, and crosslinking, while the neutral method mainly detects DSBs $[8,26,27]$. Therefore, we performed an alkaline comet assay to investigate the dose-response of the gamma-ray-induced DNA damages in rice. The '\% DNA in tail' increased somewhat dose-dependently up to $24 \mathrm{~Gy}$, but it remained constant in the range of 24 to $200 \mathrm{~Gy}$ (Figure 2). When compared with the more limited dose-dependency of the '\% DNA in tail' below 6 Gy in Arabidopsis [4], the dose-response of the '\% DNA in tail' in rice appeared greater than that of $\gamma \mathrm{H} 2 \mathrm{AX}$ below $24 \mathrm{~Gy}$. However, the relatively low confidence of the $\gamma \mathrm{H} 2 \mathrm{AX}$ assay with no $\gamma \mathrm{H} 2 \mathrm{AX}$ signal below $24 \mathrm{~Gy}$ and the saturated '\% DNA in tail' signal above 24 Gy in the alkaline comet assay demonstrated that DNA damage-based conventional methods such as $\gamma \mathrm{H} 2 \mathrm{AX}$ and comet assays may be not appropriate for phytodosimetry in rice. Moreover, the conventional models for phytodosimetry of gamma radiation showed a substantial difference in the DDR between the monocot rice and the dicot Arabidopsis. The difference in the DDR between rice and Arabidopsis may be associated with the differential expression of some DDR genes such as RPA1, BRCA1, and CYCB1;1 after gamma irradiation as previously reported [18]. 


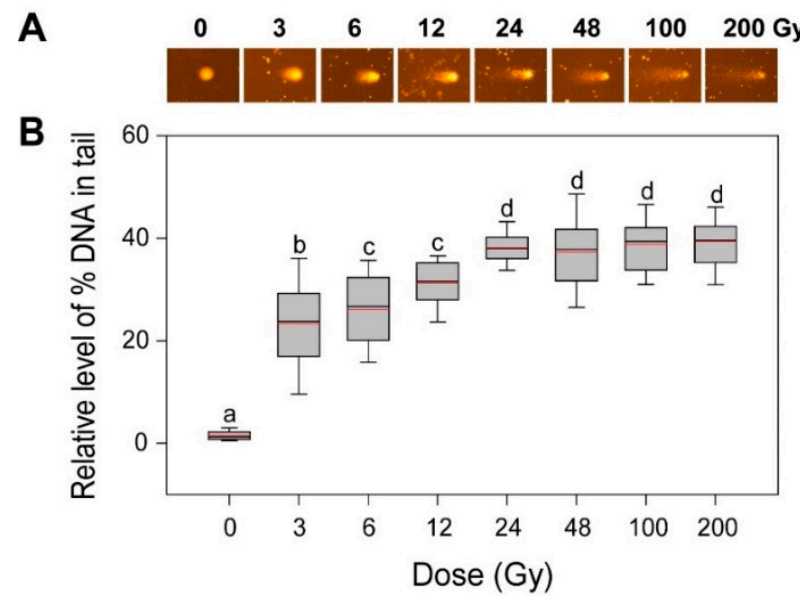

Figure 2. DNA damage in rice irradiated with different doses of gamma radiation. The representative images (A) were obtained by an alkaline comet assay and subjected to calculation for the \% DNA in tail' (B) by Komet 5.5 image analysis software (Kinetic Imaging, Ltd., Liverpool, UK). The box plots extend from the 25th to the 75th percentiles, with black and red horizontal lines at the median (50th percentile) and the mean values, respectively. Error bars indicate the 90th and 10th percentiles. Data show the distribution of 80 160 nuclei from three independent experiments. Different letters indicate significant differences at $p<0.05$ (one-way analysis of variance followed by Tukey's honestly significant difference test).

\subsection{Transcriptional Changes of Rice Gamma Ray-Responsive Genes According to the Radiation Dose}

To acquire a transcriptome-based dose-response or phytodosimetry model of gamma radiation in rice, we selected seven gamma-ray-responsive genes from the genome-wide rice transcriptome profile obtained after gamma irradiation of $300 \mathrm{~Gy}$ at a dose rate of $60 \mathrm{~Gy} \mathrm{~h}^{-1}$ for $5 \mathrm{~h}$ [18]. The seven selected genes included OsBRCA2, OsGRG, OsH2A, OsMutS, OsRAD51, OsRPA1, and OsWEE1, which showed relatively high transcriptional fold changes of $-3.64,11.09,-15.36,3.98,12.92,-9.40$, and -2.94 , respectively. OsRAD51, OsRPA1, and OsBRCA2 are expected to participate in HR repair, OsMutS in mismatch repair, and OsWEE1 in cell cycle arrest [28-33]. These were selected as DDR genes. In contrast, $\mathrm{OsH} 2 \mathrm{~A}$ encodes the histone $\mathrm{H} 2 \mathrm{~A}$ protein, and OsGRG is associated with a hypothetical protein. As previously shown by AtGRG [4], OsH2A and OsGRG were chosen for a reliable dose-response model due to their high and sustainable transcriptional changes after gamma irradiation. In the present study, the applicability and reliability of the seven gamma-rayresponsive genes were further evaluated for phytodosimetry after gamma irradiation at relatively low doses of 3 to 48 Gy using RT-PCR analysis. Gamma irradiation with 3, 6, 12, 24, or 48 Gy for $1 \mathrm{~h}$ induced dose-dependent transcriptional increases in OsRAD51, OsGRG, and OsMutS or decreases in OsRPA1 and OsWEE1, while the expressions of OsBRCA2 and $\mathrm{OsH} 2 \mathrm{~A}$ were rarely changed by the low doses of gamma radiation (Figure 3 ). These results demonstrated that the trends or intensities of transcriptional changes after gamma irradiation were different between the doses of 300 and 3 48 Gy. OsRAD51, OsGRG, OsMutS, and OsRPA1 may be more reliable gamma-ray-responsive genes in the dose range of $3 \sim 48 \mathrm{~Gy}$ than $O s B R C A 2$ and $O s H 2 A$. In addition, the transcriptional changes of OsBRCA2 and OsRPA1 in Figure 3 were discriminated from those of AtBRCA1 and AtRPA1E in our previous study [4]. The seemingly substantial difference in the transcriptional changes in the selected DDR genes between rice and Arabidopsis, probably due to their varied radiation sensitivities, makes it difficult to develop an integrated phytodosimetry model based on DDR marker genes common to both plant species. In contrast, gamma-ray-responsive and species-specific genes such as OsGRG and AtGRG encoding a different hypothetical protein may be useful in improving transcriptome-based phytodosimetry models in plants by 
correcting the differential transcriptional changes in DDR genes common to both monocot and dicot plants.

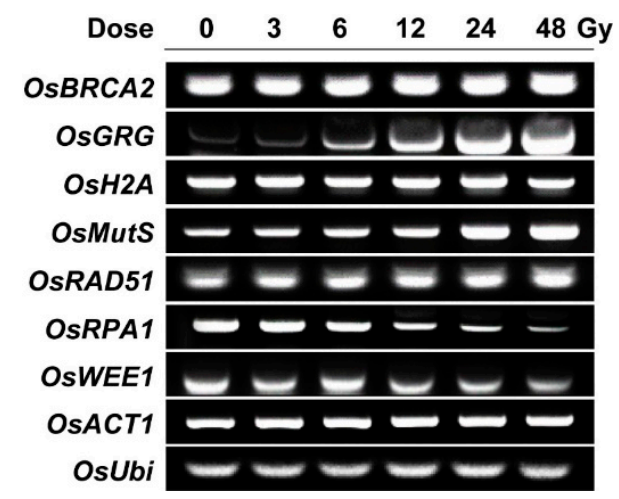

Figure 3. Transcription levels of gamma-ray-responsive genes in rice irradiated with different doses of gamma radiation. OsBRCA2, OsGRG, OsH2A, OsMutS, OsRAD51, OsRPA1, and OsWEE1 were selected as gamma ray-responsive genes from our previous study [18]. Seedlings were irradiated with gamma radiation for $1 \mathrm{~h}$ at a dose rate of 3,6,12,24, or $48 \mathrm{~Gy} \mathrm{~h}^{-1}$, and then they were subjected to the reverse transcription polymerase chain reaction (PCR) assay as described in the Materials and Method section. OsACT1 and OsUbi were used as endogenous control genes.

\subsection{Dose-Response Curves of the Selected Gamma Ray-Responsive Genes in Rice after Gamma Irradiation}

To evaluate transcriptome-based phytodosimetry models in rice, we generated quantitative dose-response curves of the selected gamma-ray-responsive genes and fitted them to different nonlinear regression equations. Gamma-ray-induced transcription of DDR genes was recently shown to depend on both the radiation dose and dose rate [4]. In the present study, various irradiation doses reflected different dose rates in $1 \mathrm{~h}$ of irradiation time. Since the radiation dose is proportional to the dose rate in a fixed irradiation time, it is unlikely to distinguish the former effects from the latter ones in biodosimetry or phytodosimetry. As expected by Figure 3, the gamma-ray-responsive transcriptional changes of OsBRCA2, $O s H 2 A$, and OsWEE1 were fitted to quadratic or exponential decay equations with a low coefficient of determination $\left(r^{2}<0.95\right)$, while those of OsGRG, OsMutS, OsRAD51, and OsRPA1 represented more reliable dose-dependent equations with $r^{2}>0.99$ (Figure 4).

This result implies that the latter four genes may be considered as marker genes for transcriptome-based phytodosimetry of gamma radiation in rice. However, these genes displayed a lack of reliable statistical difference or dose-dependency of transcriptional changes in the dose range of 3-12 Gy. Therefore, two or three dose-response curves of OsGRG, OsMutS, and OsRAD51 with a linear or supra-linear quadratic equation were integrated to compare the reliability or statistical confidence of the transcriptome-based dose-response models based on multiple genes (Figure 5). The integrated dose-response models displayed a reliable quadratic equation $\left(r^{2}>0.99\right)$ but demonstrated an increased dependency for the genes (OsGRG > OsRAD51 > OsMutS) with higher transcriptional induction. This indicates that the relative weighting or contribution of each gene in the integrated transcriptome-based dose-response model using multiple genes needs to be corrected based on the trend and amplitude of transcriptional change. In addition, the species-specific marker genes displaying distinctive transcriptional changes between rice and Arabidopsis may contribute to reliability and differentiation of transcriptome-based models in monocot and dicot plants. 

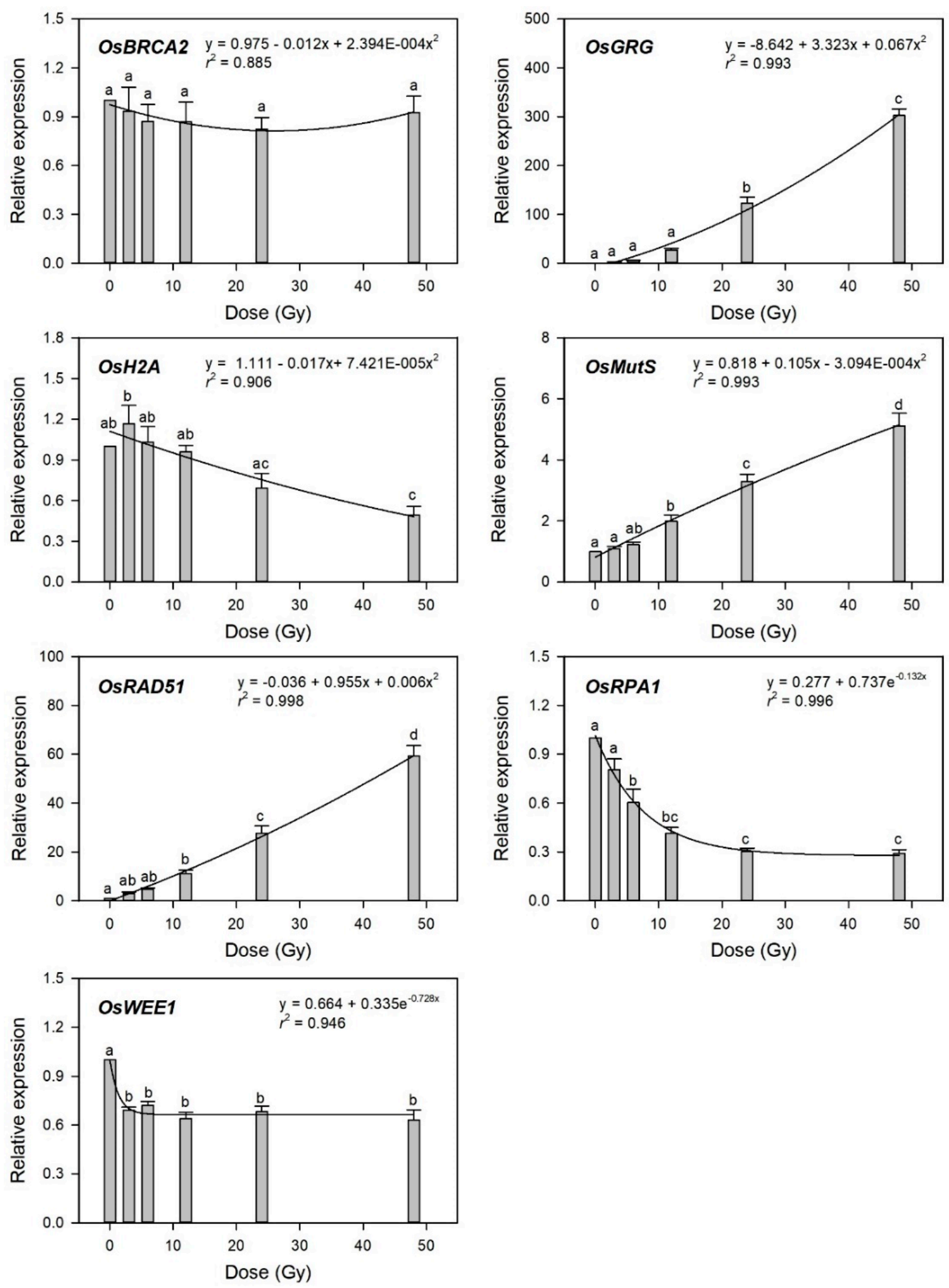

Figure 4. Relative transcription levels of the selected gamma-ray-responsive genes and dose-response curves after irradiation as measured by quantitative real-time polymerase chain reaction (RT-qPCR). All transcription levels are shown relative to the 0 Gy group of Dongjin-byeo seedlings. Data are the mean \pm SE with $n=9$ from three independent experiments. Different letters indicate significant differences at $p<0.05$ (one-way analysis of variance followed by Tukey's honestly significant difference test). 

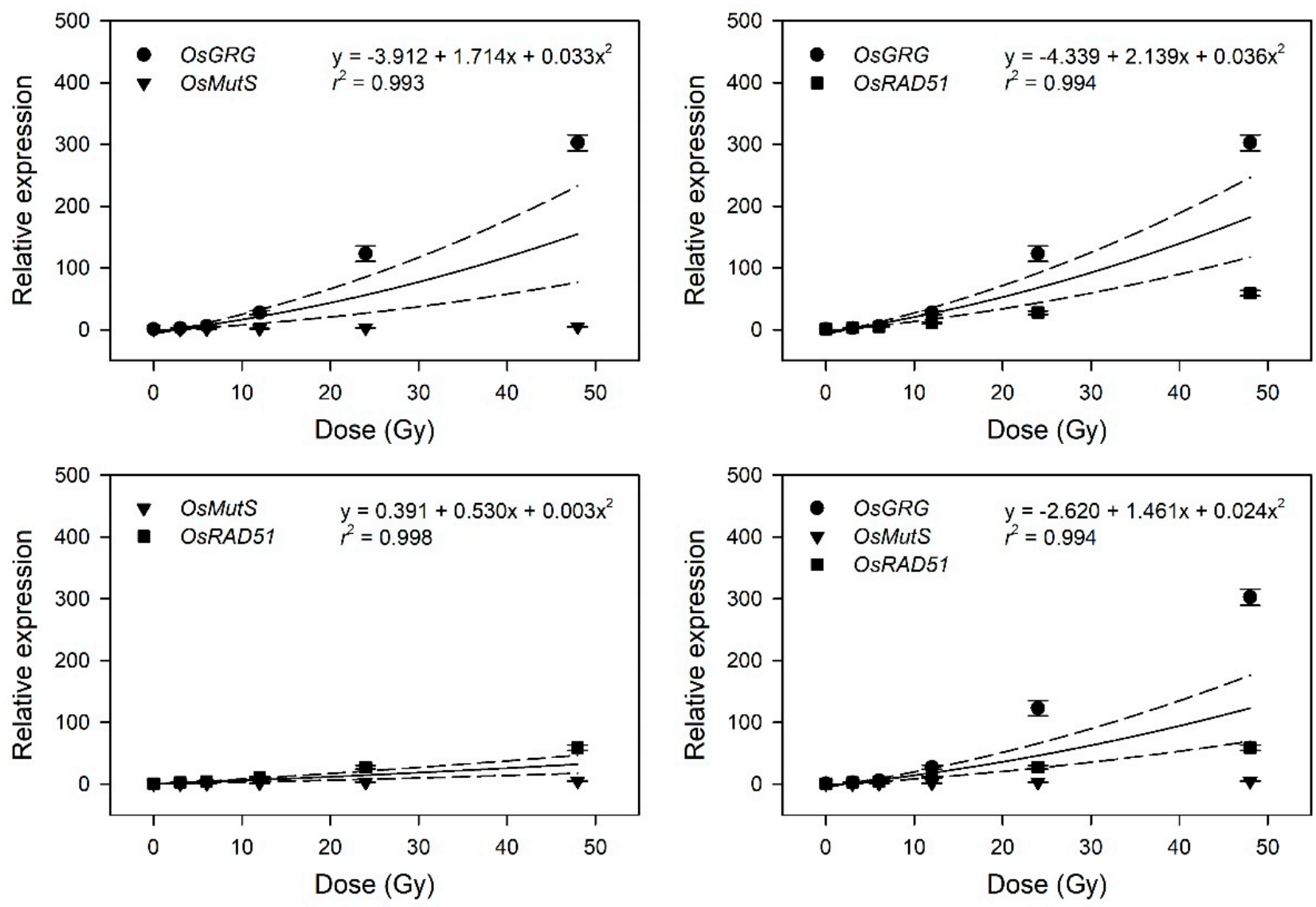

Figure 5. Dose-response curves pooled from the relative transcription levels of three selected gamma-ray-responsive genes. The dose-response curves of OsGRG, OsMutS, and OsRAD51 were integrated as a linear-quadratic equation. The solid line is the linear regression fitted line with $r^{2}$ values, and the dashed lines show the upper and lower $95 \%$ confidence intervals.

\subsection{Distinctive Genotoxicity-and Transcriptome-Based Dose-Response Curves between Rice and Arabidopsis}

The genotoxicity- and transcriptome-based dose-response curves generated by the $\gamma \mathrm{H} 2 \mathrm{AX}$, comet, and RT-qPCR assays were further compared to evaluate the relative confidence of different phytodosimetry models in rice and Arabidopsis. The dose-response curves of $\gamma \mathrm{H} 2 \mathrm{AX}$ induction displayed a high dose-dependency in Arabidopsis within the dose range of 3-48 Gy, but almost no change in rice (Figure 6A). In contrast, the olive tail moment (OTM), which was shown as the most informative parameter of the comet image for radiation-induced DNA damage in mammalian cells [34], represented a much more dose-dependent hyperbolic dose-response curve in rice compared to that in Arabidopsis (Figure 6B). This result may suggest that major types or intensities of DNA damages caused by gamma irradiation are different between rice and Arabidopsis. Compared with the two genotoxicity-based curves, the transcriptome-based dose-response curves using $R A D 51$, a representative DDR gene, commonly displayed high dose-dependency in both rice and Arabidopsis, with a greater dose-response in the former (Figure 6C). Therefore, the transcriptome-based dose-response models using the general DDR marker genes are unlikely to be strongly correlated with an actual dose-dependency of genotoxicity or DNA damage. Although these models would be more applicable for phytodosimetry due to the more reliable and higher dose-dependency, they need to be more consistent with the genotoxicity-based conventional dose-response models. 

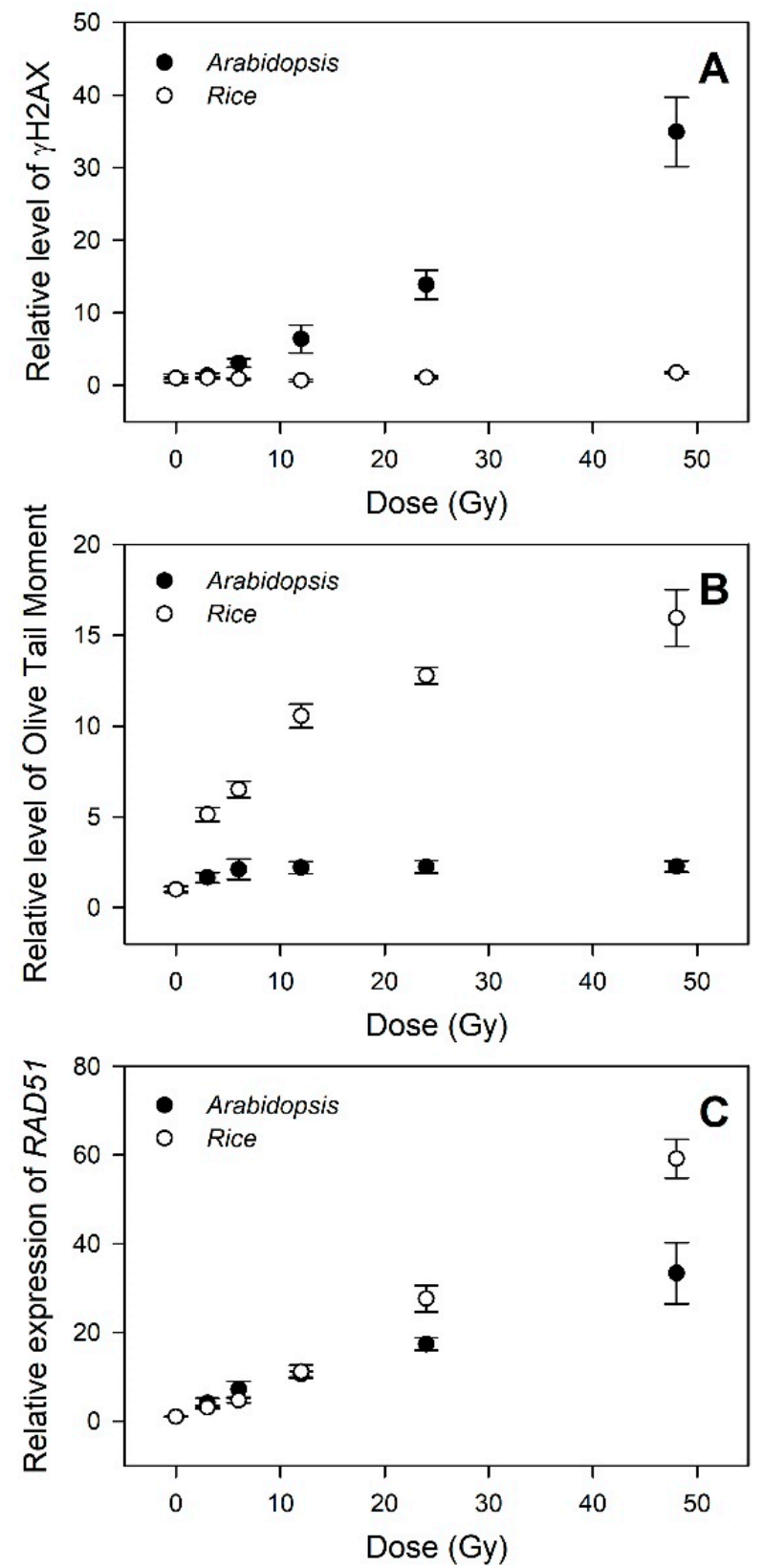

Figure 6. Difference in the DNA damage response (DDR)-based conventional and transcriptional dose-response curves between rice and Arabidopsis. (A-C) represent the relative levels of $\gamma \mathrm{H} 2 \mathrm{AX}$, olive tail moment (OTM), and RAD51 transcription, respectively. All data are shown relative to the 0 Gy group. The data for Arabidopsis were obtained from our previous study [4]. The OTM values were calculated as (Tail.mean - Head.mean) $\times$ Tail \% DNA/100 by Komet 5.5 image analysis software (Kinetic Imaging, Ltd., Liverpool, UK).

\subsection{Transcriptional Variation of the Selected Gamma-Ray-Responsive Genes in Rice and} Arabidopsis at Different Developmental Stages

In Arabidopsis, the developmental transition from the vegetative to reproductive stage caused differential physiological changes in plants irradiated with gamma radiation [35], and plants irradiated with an X-ray at different developmental stages showed distinctive expression patterns of DNA repair and epigenetic regulator genes [36]. Even without such a dramatic transition from the vegetative to reproductive stage, a specific developmental stage would differentiate the effects of ionizing radiation at the physiological and transcriptional levels in both monocot and dicot plants. Therefore, we investigated whether different 
vegetative stages or seedling ages after sowing affect the dose-dependent transcriptions of the selected gamma-ray-inducible marker genes, including OsGRG, OsMutS, OsRAD51, and OsRPA1, in rice, or AtGRG, AtPARP1, AtRAD51, and AtRPA1E in Arabidopsis used for transcriptome-based phytodosimetry models. Although OsGRG, AtGRG, and AtPARP1 seemed to be more expressed in the 20-day-old group than in the 10-day-old one, the overall transcription patterns of the genes tested were not substantially different between the two groups in both rice and Arabidopsis (Figure 7). This result suggests that the selected marker genes would have little sensitivity to such a developmental difference in the vegetative stage in both plant species, thereby supporting the reliability and statistical confidence of transcriptome-based models. However, transcriptome-based dose-response models need to be further improved by taking into account additional plant species, developmental stages, environmental factors, and genotoxicity-dependent marker genes, or by directly associating transcription with genotoxicity.
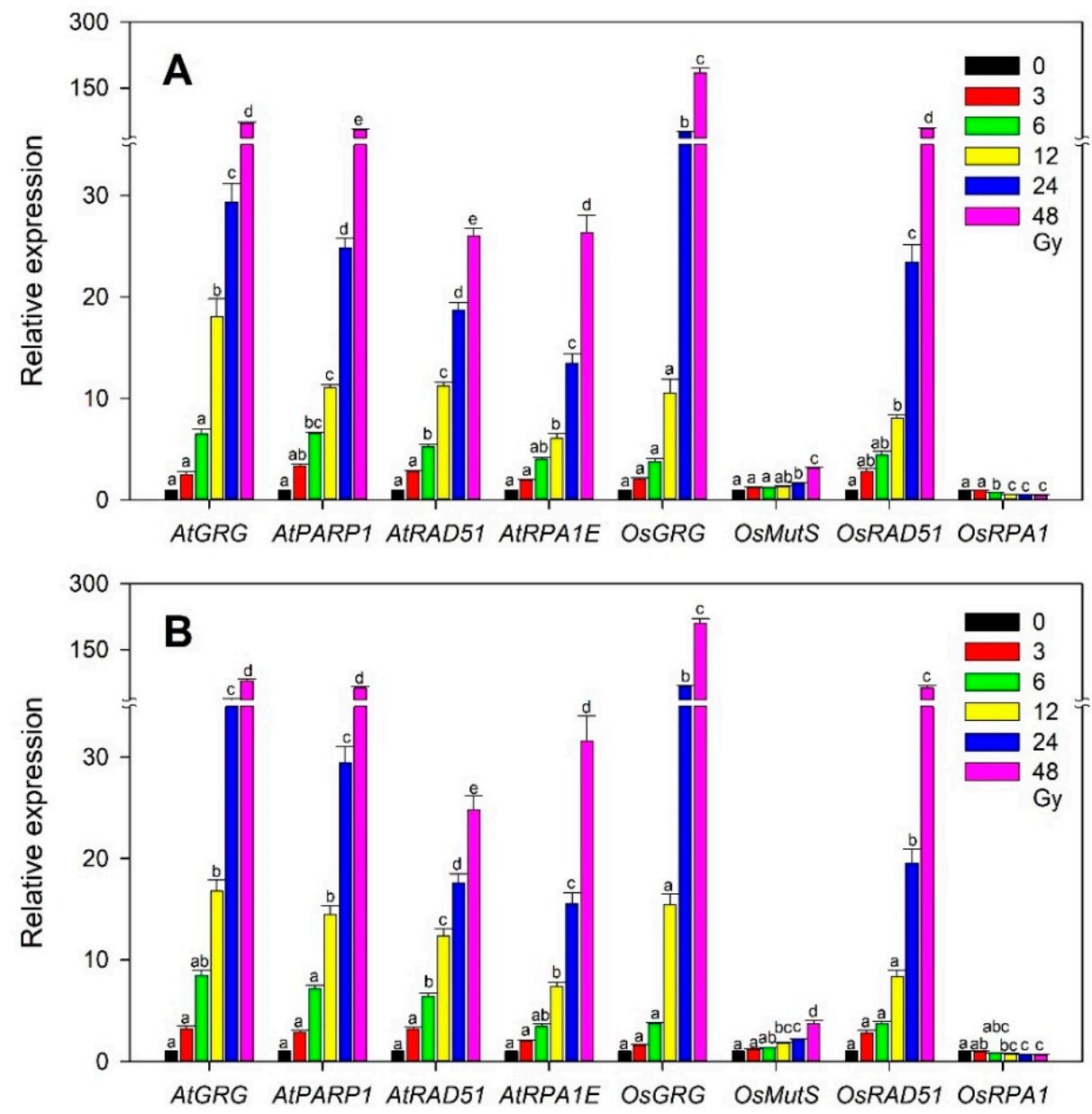

Figure 7. Difference in the dose-dependent transcription of the selected rice and Arabidopsis gammaray-responsive genes at two developmental stages or seedling ages after sowing. (A,B) represent 10- and 20-day-old seedlings, respectively. All transcription levels are shown relative to the $0 \mathrm{~Gy}$ group. Data are the mean \pm SE with $n=9$ from three independent experiments. Different letters indicate significant differences at $p<0.05$ (one-way analysis of variance followed by Tukey's honestly significant difference test).

\section{Materials and Methods}

\subsection{Plant Materials and Gamma Irradiation}

Rice seeds (Oryza sativa L. ssp. japonica cvs. Dongjin-byeo and Shindongjin-byeo) were obtained from the National Institute of Agricultural Sciences (Jeollabuk-do, Korea) and surface sterilized for 30 min with $2.5 \%$ sodium hypochlorite solution, rinsed three times 
with sterile deionized water, and incubated at $30{ }^{\circ} \mathrm{C}$ for about 2 days. Then, the seeds were subjected to hydroponic cultivation for 12 days under a $16 \mathrm{~h}$ light $/ 8 \mathrm{~h}$ dark cycle at $23^{\circ} \mathrm{C}$. Arabidopsis thaliana seeds of ecotype Columbia- 0 were shortly sterilized with $70 \%$ ethanol for $1 \mathrm{~min}$ and $20 \%$ bleach solution for $5 \mathrm{~min}$. They were cultivated on a 1/2 Murashige and Skoog (MS) medium with 1.5\% sucrose and $0.65 \%$ Phytoagar under the same photocycle and temperature regime. Arabidopsis and rice seedlings at three developmental stages (seedling ages after sowing) of 10, 14, and 20 days were irradiated with gamma radiation for $1 \mathrm{~h}$ at a dose rate of $3,6,12,24,48,100$, or $200 \mathrm{~Gy} \mathrm{~h}^{-1}$ using a $3 \mathrm{kCi}^{60} \mathrm{Co}$ source at the Advanced Radiation Technology Institute (Jeollabuk-do, Korea). Only aerial parts of the seedlings were pooled immediately after gamma irradiation, frozen with liquid nitrogen, and stored at $-80^{\circ} \mathrm{C}$. Unless otherwise noted, the subsequent $\gamma \mathrm{H} 2 \mathrm{AX}$, comet, and RT-qPCR assays were performed using 14-day-old Arabidopsis or Shindongjin-byeo seedlings.

\section{2. $\gamma H 2 A X$ and Comet Assays}

For the $\gamma \mathrm{H} 2 \mathrm{AX}$ assays, nuclear proteins were extracted as previously described [4]. To avoid protein degradation and dephosphorylation, a nuclear isolation buffer $(0.25 \mathrm{M}$ sucrose, $60 \mathrm{mM} \mathrm{KCl}, 15 \mathrm{mM} \mathrm{NaCl}, 5 \mathrm{mM} \mathrm{MgCl}, 1 \mathrm{mM} \mathrm{CaCl}$, $15 \mathrm{mM}$ 1,4-Piperazinediethanesulfonic acid (PIPES) pH 6.8, 0.8\% Triton X-100, and $1 \mathrm{mM}$ Phenylmethanesulfonyl fluoride (PMSF)) was supplemented with a protease inhibitor cocktail (cOmplete ULTRA Tablets; Roche Diagnostics, Mannheim, Germany) and a phosphatase inhibitor (50 $\mathrm{mM} \mathrm{Na}_{3} \mathrm{VO}_{4}$ and $30 \mathrm{mM}$ $\mathrm{NaF}$ ). The protein samples were subjected to $15 \%$ sodium dodecyl sulfate-polyacrylamide gel electrophoresis, blotted, and immunodetected with rabbit anti-human $\gamma \mathrm{H} 2 \mathrm{AX}$ antibodies (1:1000; Sigma-Aldrich, St. Louis, MO, USA) as previously described [4,24,37]. The band intensities on the immunoblots were determined using ImageJ $1.52 \mathrm{v}(\mathrm{NIH}$, Bethesda, MD, USA).

For the comet assays, nuclei were obtained by slicing aerial tissues with a razor blade in $1 \times$ phosphate-buffered saline supplemented with $50 \mathrm{mM}$ Ethylenediaminetetraacetic acid (EDTA) on ice [4]. The nuclei were collected by centrifugation and fixed in $1 \%$ low melting point agarose on microscope slides precoated with $1 \%$ normal melting point agarose. They were then subjected to unwinding in a high alkaline buffer $(0.3 \mathrm{M} \mathrm{NaOH}, 5 \mathrm{mM}$ EDTA $\mathrm{pH}>13.0$ ) and electrophoresis in the same solution followed by neutralization in $100 \mathrm{mM}$ Tris- $\mathrm{HCl}$. The slide samples were sequentially washed with $1 \%$ Triton $\mathrm{X}-100$ followed by $70 \%$ and $96 \%$ ethanol, and then stained with propidium iodide solution $\left(2.5 \mu \mathrm{g} \mathrm{mL}^{-1}\right)$. The microscopic images were analyzed using Komet 5.5 image analysis software (Kinetic Imaging, Ltd., Liverpool, UK).

\subsection{Quantitative Real-Time Polymerase Chain Reaction (RT-qPCR) Analysis}

Total RNA was extracted from 10-, 14-, or 20-day-old seedlings using a RNeasy Plant Mini Kit (QIAGEN, Hilden, Germany) and cDNA was synthesized from $1 \mu \mathrm{g}$ of each RNA sample using oligo(dT) primers and a LaboPass ${ }^{\mathrm{TM}}$ cDNA Synthesis Kit (Cosmogenetech, Seoul, Korea). The subsequent PCR was performed in a LaboPass ${ }^{\mathrm{TM}}$ IP pro-Taq PCR Mastermix (Cosmogenetech) with 26-30 cycle reactions of $95^{\circ} \mathrm{C}$ for $10 \mathrm{~s}, 58{ }^{\circ} \mathrm{C}$ for $10 \mathrm{~s}$, and $72{ }^{\circ} \mathrm{C}$ for $1 \mathrm{~min}$ using the gene-specific primers (Table 1 ). In contrast, the quantitative PCR (qPCR) amplifications were performed at $95^{\circ} \mathrm{C}$ for $30 \mathrm{~s}$, followed by 40 cycles of $95^{\circ} \mathrm{C}$ for $10 \mathrm{~s}, 58^{\circ} \mathrm{C}$ for $10 \mathrm{~s}$, and $72{ }^{\circ} \mathrm{C}$ for 1 min with a CFX Connect ${ }^{\mathrm{TM}}$ Real-Time PCR Detection System (Bio-Rad Laboratories, Hercules, CA, USA) using an iTaq Universal SYBR ${ }^{\circledR}$ Green Supermix (Bio-Rad Laboratories). The amount of cDNA was $1 \mu \mathrm{L}$ for PCR and $2 \mu \mathrm{L}$ for qPCR. The relative expression level of each gene was calculated between the control and gamma-irradiated samples using the comparative $C_{T}$ method [38]. The relative mRNA expression data of the three biological replicates in the $\mathrm{QPCR}$ were normalized against the reference gene OsUBi for rice or AtACT2 for Arabidopsis. OsUBi, OsACT1 and AtACT2 genes were used as endogenous controls to normalize for differences in the amount of total RNA. 
Table 1. Primer sequences used for RT-qPCR. Parentheses represent Rice the Annotation Project Database (RAPdb) or Arabidopsis Genome Initiative (AGI) numbers. OsUbi and OsACT1 in rice, and AtACT2 in Arabidopsis were used as endogenous controls. OsGRG and AtGRG represent for Oryza sativa Gamma-ray Responsive Gene and Arabidopsis thaliana Gamma-ray Responsive Gene, respectively.

\begin{tabular}{|c|c|}
\hline Gene & Primer Sequence (Forward/Reverse) \\
\hline OsACT1 (Os03g0718100) & 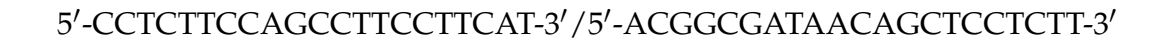 \\
\hline OsBRCA2 (Os01g0164900) & 5'-GCAAAATGAAGTAGCTAAGAAG-3' /5'-GTCTGTGCGGTTGCTAAAGG-3' \\
\hline OsGRG (Os04g0403400) & 5'-CTACTGAAGCCAGAGCCGTTTC-3' / $5^{\prime}$-CTAACGATGTCGCAGGCCTATC-3' \\
\hline OsH2A (Os03g0279200) & 5'-GCCGGGAAGTCCCCCAAGAAG-3' / $5^{\prime}$-GACACAAGCACAGATCACAAGG-3' \\
\hline OsMutS (Os05g0498300) & 5'-ACTTGGTTGGAAAGGCCAATTC-3' /5'-TTCATTGGCTGACACCTGCTC-3' \\
\hline OsRAD51 (Os12g0497300) & 5'-CTTCAGGATACAGCATGAGTTTGC-3' /5'-GTACACCCCCGCTGAAACAC-3' \\
\hline OsRPA1 (Os03g0214100) & 5'-GTTCTCTCCAAGCCCACGAAC-3' / 5'-TTGTACGTCCTCAGGTTGCC-3' \\
\hline OsUbi (Os01g0328400) & 5'-ACCACTTCGACCGCCACTACT-3' /5'-ACGCCTAAGCCTGCTGGTT-3' \\
\hline OsWEE1 (Os02g0135300) & 5'-CCATCTGCGAAAGAAGTCCTG-3' / 5'-TTGGGGAGTTTCTCTTGGTG-3' \\
\hline AtACT2 (At3g18780) & 5'-GCCCAGAAGTCTTGTTCCA-3' /5'-CTTGGTGCAAGTGCTGTGAT-3' \\
\hline AtGRG (At4g22960) & 5'-AGGGTACAAAAGGGCTCACG-3' /5'-TGCGGAACAGGACACAAAGT-3' \\
\hline AtRAD51 (At5g20850) & 5'-TACCGCTCTCTACAGAACAG-3' /5'-ATTCTCTCCTCTGCTCTTCC-3' \\
\hline AtRPA1E (At4g19130) & 5'-TGGAGAAGTGACGACTGAAGC-3' / 5'-ACCTCCAGTTGCGGAACAAT-3' \\
\hline AtPARP1 (At2g31320) & 5'-ACCCATCAGAGGCTCAAACA-3' / 5' -ACGCATCTTGATTTGTTCCACA-3' \\
\hline
\end{tabular}

\subsection{Statistical Analysis}

All experiments were repeated three times using the biological replicates harvested after different gamma irradiations. The data were subjected to statistical nonlinear (polynomial) regression analysis and/or one-way analysis of variance followed by Tukey's honest significance difference test using the statistical and graphical functions of SigmaPlot 12.0 and PASW Statistics 18 (SPSS, Chicago, IL, USA). A p-value less than 0.05 was considered significant.

\section{Conclusions}

Currently, transcriptome data are widely used in molecular diagnostics of environmental threats to plants. Species-specific and genome-wide transcriptomic profiles are easily available through RNA-Seq analysis and are applicable to reliable and fast evaluation of stress-induced responses in plants. In this context, our study shows that transcriptomebased phytodosimetry models could be reliable in both monocot and dicot plants as shown in rice and Arabidopsis. However, the distinctive genotoxicity- or transcriptome-based models between rice and Arabidopsis demonstrated that phytodosimetry of genotoxicity would depend on the relative toxicity specific to a species or an individual. Therefore, since transcriptome-based models are reliable only with their representative materials, additional models using regional representative plant species will help to improve environmental risk assessment of ionizing radiation. In contrast, the effective integration of multiple transcriptome-based models from various plant species needs to be further studied to develop a standardized model of phytodosimetry that is applicable to a broad range of environmental regions.

Author Contributions: Conceptualization, J.-H.K.; methodology, J.-H.K., M.-S.C., and B.-Y.C.; validation, J.-H.K., B.-Y.C., and S.L.; formal analysis and investigation, J.-H.K., K.H., E.L., and S.K.D.; resources, J.-H.K. and M.-S.C.; data curation, J.-H.K., K.H., and E.L.; writing-original draft preparation, J.-H.K.; writing—review and editing, J.-H.K., M.-S.C., and S.L.; visualization; J.-H.K. and K.H.; supervision, J.-H.K.; project administration and funding acquisition, S.L. All authors have read and agreed to the published version of the manuscript. 
Funding: This study was supported by the research program of the Korea Atomic Energy Research Institute funded by the Ministry of Science and ICT, Republic of Korea.

Institutional Review Board Statement: Not applicable.

Informed Consent Statement: Not applicable.

Data Availability Statement: Not applicable.

Conflicts of Interest: The authors declare no conflict of interest.

\section{References}

1. Dinis, M.L.; Fiúza, A. Exposure assessment to radionuclides transfer in food chain. In Multiple Stressors: A Challenge for the Future; Mothersill, C., Mosse, I., Seymour, C., Eds.; Springer: Dordrecht, The Netherlands, 2007; pp. 309-323.

2. IAEA. Environmental and Source Monitoring for Purposes of Radiation Protection; International Atomic Energy Agency: Vienna, Austria, 2005.

3. ICRP. Environmental Protection: The Concept and Use of Reference Animals and Plants, ICRP Publication 108. Ann. ICRP 2008, $38,4-6$.

4. Ryu, T.H.; Kim, J.K.; Kim, J.I.; Kim, J.-H. Transcriptome-based biological dosimetry of gamma radiation in Arabidopsis using DNA damage response genes. J. Environ. Radioactiv. 2018, 181, 94-101. [CrossRef] [PubMed]

5. Pernot, E.; Hall, J.; Baatout, S.; Benotmane, M.A.; Blanchardon, E.; Bouffler, S.; El Saghire, H.; Gomolka, M.; Guertler, A.; HarmsRingdahl, M.; et al. Ionizing radiation biomarkers for potential use in epidemiological studies. Mutat. Res. 2012, 751, 258-286. [CrossRef]

6. Rodrigues, G.S.; Ma, T.-H.; Pimentel, D.; Weinstein, L.H. Tradescantia bioassays as monitoring systems for environmental mutagenesis: A review. Crit. Rev. Plant Sci. 1997, 16, 325-359. [CrossRef]

7. IAEA. Cytogenetic Dosimetry: Applications in Preparedness for and Response to Radiation Emergencies; International Atomic Energy Agency: Vienna, Austria, 2011.

8. Garaj-Vrhovac, V.; Kopjar, N.; Razem, D.; Vekic, B.; Miljanic, S.; Ranogajec-Komor, M. Application of the alkaline comet assay in biodosimetry: Assessment of in vivo DNA damage in human peripheral leukocytes after a gamma radiation incident. Radiat. Prot. Dosim. 2002, 98, 407-416. [CrossRef] [PubMed]

9. Horn, S.; Barnard, S.; Rothkamm, K. Gamma-H2AX-based dose estimation for whole and partial body radiation exposure. PLoS ONE 2011, 6, e25113. [CrossRef]

10. Touil, N.; Aka, P.V.; Buchet, J.P.; Thierens, H.; Kirsch-Volders, M. Assessment of genotoxic effects related to chronic low level exposure to ionizing radiation using biomarkers for DNA damage and repair. Mutagenesis 2002, 17, 223-232. [CrossRef]

11. Zeegers, D.; Venkatesan, S.; Koh, S.W.; Low, G.K.; Srivastava, P.; Sundaram, N.; Sethu, S.; Banerjee, B.; Jayapal, M.; Belyakov, O.; et al. Biomarkers of Ionizing Radiation Exposure: A Multiparametric Approach. Genome Integr. 2017, 8, 6. [CrossRef]

12. Hall, J.; Jeggo, P.A.; West, C.; Gomolka, M.; Quintens, R.; Badie, C.; Laurent, O.; Aerts, A.; Anastasov, N.; Azimzadeh, O.; et al. Ionizing radiation biomarkers in epidemiological studies-An update. Mutat. Res. 2017, 771, 59-84. [CrossRef]

13. Kim, J.-H.; Kim, J.E.; Lee, M.H.; Lee, S.W.; Cho, E.J.; Chung, B.Y. Integrated analysis of diverse transcriptomic data from Arabidopsis reveals genetic markers that reliably and reproducibly respond to ionizing radiation. Gene 2013, 518, $273-279$. [CrossRef] [PubMed]

14. Wang, W.; Scheffler, K.; Esbensen, Y.; Eide, L. Quantification of DNA Damage by Real-Time qPCR. Methods Mol. Biol. 2016, 1351, 27-32. [CrossRef]

15. Nelson, D.R.; Schuler, M.A.; Paquette, S.M.; Werck-Reichhart, D.; Bak, S. Comparative genomics of rice and Arabidopsis. Analysis of 727 cytochrome P450 genes and pseudogenes from a monocot and a dicot. Plant Physiol. 2004, 135, 756-772. [CrossRef] [PubMed]

16. Paterson, A.H.; Bowers, J.E.; Chapman, B.A.; Peterson, D.G.; Rong, J.; Wicker, T.M. Comparative genome analysis of monocots and dicots, toward characterization of angiosperm diversity. Curr. Opin. Biotechnol. 2004, 15, 120-125. [CrossRef]

17. Wu, Y.; Ma, X.; Pan, Z.; Kale, S.D.; Song, Y.; King, H.; Zhang, Q.; Presley, C.; Deng, X.; Wei, C.I.; et al. Comparative genome analyses reveal sequence features reflecting distinct modes of host-adaptation between dicot and monocot powdery mildew. BMC Genomics 2018, 19, 705. [CrossRef]

18. Kim, J.-H. Functional characteristics of genome-wide rice transcriptomes responded to gamma radiation. J. Radiat. Ind. 2019, $13,55-60$.

19. Esnault, M.-A.; Legue, F.; Chenal, C. Ionizing radiation: Advances in plant response. Environ. Exp. Bot. 2010, 68, 231-237. [CrossRef]

20. Zheng, Y.; Sanche, L. Clustered DNA Damages induced by 0.5 to $30 \mathrm{eV}$ Electrons. Int. J. Mol. Sci. 2019, 20, 3749. [CrossRef]

21. Kim, J.H.; Ryu, T.H.; Lee, S.S.; Lee, S.; Chung, B.Y. Ionizing radiation manifesting DNA damage response in plants: An overview of DNA damage signaling and repair mechanisms in plants. Plant Sci. 2019, 278, 44-53. [CrossRef]

22. Kim, J.-H.; Chung, B.Y.; Kim, J.-S.; Wi, S.G. Effects of in planta gamma-irradiation on growth, photosynthesis, and antioxidative capacity of red pepper (Capsicum annuum L.) plants. J. Plant Biol. 2005, 48, 47-56. [CrossRef] 
23. Kim, J.-H.; Moon, Y.R.; Lee, M.H.; Chung, B.Y. Change of chlorophyll fluorescence transients in Arabidopsis plants irradiated with low-dose radiation using a gamma phytotron. Int. J. Low Radiat. 2010, 7, 253-258. [CrossRef]

24. Choi, S.H.; Ryu, T.H.; Kim, J.I.; Lee, S.; Lee, S.S.; Kim, J.H. Mutation in DDM1 inhibits the homology directed repair of double strand breaks. PLoS ONE 2019, 14, e0211878. [CrossRef]

25. Friesner, J.D.; Liu, B.; Culligan, K.; Britt, A.B. Ionizing radiation-dependent gamma-H2AX focus formation requires ataxia telangiectasia mutated and ataxia telangiectasia mutated and Rad3-related. Mol. Biol. Cell 2005, 16, 2566-2576. [CrossRef] [PubMed]

26. Menke, M.; Chen, I.; Angelis, K.J.; Schubert, I. DNA damage and repair in Arabidopsis thaliana as measured by the comet assay after treatment with different classes of genotoxins. Mutat. Res. 2001, 493, 87-93. [CrossRef]

27. Georgieva, M.; Rashydov, N.M.; Hajduch, M. DNA damage, repair monitoring and epigenetic DNA methylation changes in seedlings of Chernobyl soybeans. DNA Repair 2017, 50, 14-21. [CrossRef] [PubMed]

28. Aklilu, B.B.; Soderquist, R.S.; Culligan, K.M. Genetic analysis of the Replication Protein A large subunit family in Arabidopsis reveals unique and overlapping roles in DNA repair, meiosis and DNA replication. Nucleic Acids Res. 2014, 42, 3104-3118. [CrossRef]

29. Chang, Y.; Gong, L.; Yuan, W.; Li, X.; Chen, G.; Li, X.; Zhang, Q.; Wu, C. Replication protein A (RPA1a) is required for meiotic and somatic DNA repair but is dispensable for DNA replication and homologous recombination in rice. Plant Physiol. 2009, 151, 2162-2173. [CrossRef]

30. Culligan, K.M.; Hays, J.B. Arabidopsis MutS homologs-AtMSH2, AtMSH3, AtMSH6, and a novel AtMSH7-form three distinct protein heterodimers with different specificities for mismatched DNA. Plant Cell 2000, 12, 991-1002. [CrossRef]

31. De Schutter, K.; Joubes, J.; Cools, T.; Verkest, A.; Corellou, F.; Babiychuk, E.; Van Der Schueren, E.; Beeckman, T.; Kushnir, S.; Inze, D.; et al. Arabidopsis WEE1 kinase controls cell cycle arrest in response to activation of the DNA integrity checkpoint. Plant Cell 2007, 19, 211-225. [CrossRef]

32. Rajanikant, C.; Melzer, M.; Rao, B.J.; Sainis, J.K. Homologous recombination properties of OsRad51, a recombinase from rice. Plant Mol. Biol. 2008, 68, 479-491. [CrossRef]

33. Seeliger, K.; Dukowic-Schulze, S.; Wurz-Wildersinn, R.; Pacher, M.; Puchta, H. BRCA2 is a mediator of RAD51- and DMC1facilitated homologous recombination in Arabidopsis thaliana. N. Phytol. 2012, 193, 364-375. [CrossRef]

34. Olive, P.L.; Banath, J.P.; Durand, R.E. Heterogeneity in radiation-induced DNA damage and repair in tumor and normal cells measured using the "comet" assay. 1990. Radiat. Res. 2012, 178, AV35-AV42. [CrossRef] [PubMed]

35. Kim, J.-H.; Moon, Y.R.; Lee, M.H.; Kim, J.H.; Wi, S.G.; Park, B.J.; Kim, C.S.; Chung, B.Y. Photosynthetic capacity of Arabidopsis plants at the reproductive stage tolerates gamma irradiation. J. Radiat. Res. 2011, 52, 441-449. [CrossRef] [PubMed]

36. Sidler, C.; Li, D.; Kovalchuk, O.; Kovalchuk, I. Development-dependent expression of DNA repair genes and epigenetic regulators in Arabidopsis plants exposed to ionizing radiation. Radiat. Res. 2015, 183, 219-232. [CrossRef]

37. Song, J.; Bent, A.F. Microbial pathogens trigger host DNA double-strand breaks whose abundance is reduced by plant defense responses. PLoS Pathog. 2014, 10, e1004030. [CrossRef]

38. Liu, W.; Saint, D.A. A new quantitative method of real time reverse transcription polymerase chain reaction assay based on simulation of polymerase chain reaction kinetics. Anal. Biochem. 2002, 302, 52-59. [CrossRef] 